guientes 4 meses, atenuándose paulatinamente hasta casi desaparecer en el octavo mes. A largo plazo, el efecto de la nueva infección dependió de la edad a la que se detectó la infección por primera vez y se observó un efecto acumulativo a medida que pasaba el tiempo. La talla perdida no se recuperó y al llegar al octavo mes, la diferencia acumulada era de 0,14 cm (intervalo de confianza de $95 \%$ [IC95\%]: 0,11 a 0,17 cm) en relación con los niños sin la infección. Debido a que los niños menores crecen más rápidamente, cuando la infección ocurrió antes de los 12 meses de edad, los niños perdieron en promedio $0,25 \mathrm{~cm}$ de talla en los primeros 8 meses de infección (IC95\%: 0,21 a 0,29 cm).

Con relación al peso, se encontró una pérdida de peso pequeña pero significativa inmediatamente después de establecerse la infección, aunque desapareció a la siguiente visita de control. Sin embargo, no se observó un incremento compensatorio en los siguientes meses. De esta forma, se comprobó que el efecto de la infección con $H$. pilori sobre el peso del niño es menor y de menos duración que sobre su estatura. Esto puede deberse a la naturaleza crónica de esta infección y a que el peso presenta por lo general una mayor variabilidad.

Los resultados de este trabajo indican que el efecto de la infección con $H$. pylori sobre la talla de los niños de edad preescolar es mayor inmediatamente después de la infección y decrece aproximadamente a la mitad a los 8 meses de seguimiento. El retraso del crecimiento fue acumulativo y no se recuperó, por lo que la diferencia se mantuvo al menos durante todo el período de seguimiento. El retraso acumulado del crecimiento puede deberse a las altas tasas de reinfección en algunos niños. La aplicación de medidas dirigidas a prevenir y eliminar esta infección en niños de edad preescolar puede evitar retrasos del crecimiento. (Mera RM, et al. Effects of a new Helicobacter pylori infection on height and weight in Colombian children. Ann Epidemiol. 2006;16:347-51.)

\section{Factores asociados con el estado nutricional en niños de 0 a 23 meses en Nicaragua}

Nicaragua es un país pobre con una alta prevalencia de enfermedades infecciosas y desnutrición. En este artículo se examina el estado nutricional de los niños de 0 a 23 meses en varias zonas urbanas y rurales de Nicaragua y se identifican los factores asociados con la desnutrición infantil en el país.

Se realizó un estudio transversal en el municipio de Nadine, provincia de Granada, Nicaragua, entre febrero y marzo de 2002. Se tomaron las medidas antropométricas (peso y talla) de 756 niños y se aplicó un cuestionario estructurado a sus madres. En el cuestionario se preguntó acerca de los episodios de fiebre y las enfermedades diarreicas y respiratorias que habían padecido los niños en los 14 días previos a la encuesta. Como indicadores del estado nutricional se calcularon las puntuaciones $Z$ ( $Z$ scores) de la relación peso-edad (ZPE), de la relación talla-edad (ZTE) y de la relación peso-talla (ZPT).

La proporción de niños con bajo peso (ZPE $<-2$ desviaciones estándar [DE]) fue de 10,3\%, mientras que $30,1 \%$ de los niños tenían retraso del crecimiento (ZTE $<-2 \mathrm{DE})$ y $5,0 \%$ presentaban atrofia $(\mathrm{ZPT}<-2 \mathrm{DE})$.

Se encontraron diferencias significativas $(P<$ $0,05)$ entre la ZPE de los niños de zonas urbanas $(0,37 ; \mathrm{DE}=1,26)$ y rurales $(-0,54 ; \mathrm{DE}=1,27)$. La ZTE promedio fue de $-1,01$ ( $\mathrm{DE}=1,66)$ en las zonas urbanas y de $-1,36(\mathrm{DE}=1,56)$ en las rurales $(P<0,05)$. Los niños de zonas rurales estaban más propensos a tener retraso en el crecimiento $(P<0,05)$. No se encontraron diferencias significativas entre las ZPT de los niños de las zonas urbanas y rurales (5,8 y 4,1, respectivamente).

Los factores de riesgo de retraso en el crecimiento fueron el tener una madre analfabeta o que no había recibido educación institucional (razón de posibilidades [odds ratio, RR] $=3,476 ; P<0,01$ ), haber sufrido alguna infección respiratoria $(\mathrm{RR}=$ 1,821; $P<0,05)$ o haber tenido bajo peso ( $<3000 \mathrm{~g})$ al nacer $(\mathrm{RR}=1,704 ; P<0,05)$. Los factores de riesgo del bajo peso fueron haber tenido alimentación materna por más de 12 meses $(R R=2,031 ; P<0,01)$, no haber tenido seguimiento del crecimiento $(\mathrm{RR}=$ $1,956 ; P<0,05)$ y ser de sexo femenino $(\mathrm{RR}=1,884$; $P<0,05)$.

Según los resultados del análisis bifactorial, los factores asociados con el retraso del crecimiento (ZTE $<-2$ DE) fueron: bajo ingreso mensual $(P<$ $0,01)$, tener una madre analfabeta o sin educación institucionalizada $(P<0,01)$, haber recibido alimentación materna por más de 12 meses $(P<0,01)$, vivir en la ciudad $(P<0,05)$, ser de sexo femenino $(P<$ $0,05)$, haber nacido con bajo peso $(P<0,001)$ y haber sufrido alguna infección respiratoria $(P<0,05)$. El retraso del crecimiento estuvo asociado con las condiciones socioeconómicas de la familia más que con la conducta materna de garantizar la salud, como mantener el seguimiento del crecimiento de su hijo o participar en actividades de educación sanitaria, entre otras.

En cuanto al bajo peso (ZPE <-2 DE), según el análisis bifactorial, los factores de riesgo fueron: la lactancia materna por más de 12 meses $(P<0,01)$, ser de sexo femenino $(P<0,01)$, haber nacido con bajo peso $(P<0,05)$ y no haber tenido seguimiento del crecimiento $(P<0,01)$. 
A pesar del sostenido desarrollo económico de Nicaragua a partir de 1990, el estado nutricional de los niños no ha mejorado como se esperaba. Este estudio demuestra que las condiciones socioeconómicas favorables de las madres y su participación activa en actividades de promoción de salud, tales como los programas de seguimiento del crecimiento de sus hijos, pueden proteger contra la desnutrición infantil, específicamente contra el bajo peso. (Sakisaka K, et al. Nutritional status and associated factors in children aged 0-23 months in Granada, Nicaragua. Public Health. 2006;120(5): 400-11.)

\section{Asocian la reinfección materna por Trypanosoma cruzi con una mayor morbilidad y mortalidad por enfermedad de Chagas congénita}

En América Latina, alrededor de 17 millones de personas están infectadas con el parásito Trypanosoma cruzi, agente causante de la enfermedad de Chagas. La transmisión al feto puede ocurrir hasta en $12 \%$ de los casos de madres infectadas.

El objetivo de este trabajo fue determinar el papel que desempeña la densidad vectorial (DV) del entorno materno en la parasitemia de las mujeres embarazadas y en el pronóstico de la infección congénita.

Los datos hematológicos y parasitológicos de los recién nacidos de madres infectadas con T. cruzi se compararon y estratificaron de acuerdo con la DV en la zona de residencia de las madres que dieron a luz en el hospital de maternidad Germán Urquidi, perteneciente al Hospital Universitario Vietma, de la Universidad Mayor de San Simón, en Cochabamba, Bolivia. En total se estudió a 71 niños infectados congénitamente $(\mathrm{M}+\mathrm{H}+)$ y 578 niños no infectados nacidos de madres infectadas $(\mathrm{M}+\mathrm{H}-)$ procedentes de los departamentos bolivianos de Cochabamba, Chuquisaca y Tarija, zonas con enfermedad de Chagas endémica. Se definió como caso de infección congénita la detección del parásito vivo en la sangre del recién nacido.

Entre $27 \%$ y $47 \%$ de los casos $\mathrm{M}+\mathrm{H}+$ nacidos de madres que vivían en zonas de alta DV presentaron índices de Apgar bajos $(<7)$, peso bajo al nacer $(<2500 \mathrm{~g})$, prematurez (edad gestacional $<37$ semanas) o prematurez con bajo peso. Solo se encontraron estos trastornos en $7-17 \%$ de los $\mathrm{M}+\mathrm{H}+$ cuyas madres vivían en zonas de mediana DV y en $0-8 \%$ de los $\mathrm{M}+\mathrm{H}+$ de madres que vivían en zonas de baja DV. Ningún $\mathrm{M}+\mathrm{H}+$ de madres que vivían en zonas sin vectores presentó estos trastornos. La prueba de la ji al cuadrado demostró que esta tendencia era altamente significativa $(0,05>P>0,0001)$.

Los trastornos estudiados se observaron en 3 a $11 \%$ de los $\mathrm{M}+\mathrm{H}-$, pero no se encontró asocia- ción alguna con la DV en la zona de residencia de la madre $(P>0,05)$. Al comparar los resultados de los casos $\mathrm{M}+\mathrm{H}+$ con los $\mathrm{M}+\mathrm{H}-$ nacidos de madres que vivían en zonas de alta DV, se encontró una diferencia altamente significativa $(0,01>P>0,0001)$, mientras que las comparaciones de los resultados de los casos $\mathrm{M}+\mathrm{H}+$ y $\mathrm{M}+\mathrm{H}-$ de madres que vivían en zonas de DV mediana, baja o nula no mostraron diferencias significativas $(P>0,05)$.

Estos resultados demuestran que la alta DV en la zona de residencia de la madre está asociada con una mayor frecuencia de hemocultivos maternos positivos y con formas clínicas más graves de enfermedad de Chagas congénita. Los casos $\mathrm{M}+\mathrm{H}+$ presentaron con mayor frecuencia índices de Apgar < 7 (a 1 minuto de nacidos), peso bajo al nacer, prematurez y prematurez con bajo peso. Además, la enfermedad se manifestó más grave y se acompañó de una mayor tasa de letalidad. Si embargo, no se encontró una asociación significativa entre una alta DV en el entorno materno y los valores bajos de hematócrito o hemoglobina de las madres o la morbilidad o mortalidad de los casos $\mathrm{M}+\mathrm{H}-$.

En conclusión, las frecuentes picadas de los insectos vectores de T. cruzi durante el embarazo no provocan anemia en las mujeres, pero la reinfección múltiple de la madre aumenta su parasitemia y conduce a una forma más grave de enfermedad congénita. La permanencia de futuras madres en zonas de alta DV está asociada con un alto riesgo de que los recién nacidos sufran enfermedad de Chagas congénita grave o mueran. Estos resultados respaldan la necesidad de llevar a cabo programas de lucha antivectorial en las zonas endémicas, lo que traerá como resultado una reducción de la morbilidad y la mortalidad por infección congénita con T. cruzi. (Torrico F, et al. Are maternal re-infections with Trypanosoma cruzi associated with higher morbidity and mortality of congenital Chagas disease? Trop Med Int Health. 2006; 11(5): 628-35.)

\section{Evalúan programa para la prevención de enfermedades de transmisión sexual y embarazo precoz en escuelas mexicanas}

Los intentos más recientes por disminuir la incidencia de enfermedades de transmisión sexual y sida y de reducir el número de embarazos en adolescentes se han basado en proyectos escolares que promueven el uso de condones y la abstinencia sexual. Sin embargo, los resultados de recientes metaanálisis demuestran que estas estrategias no han sido evaluadas con el rigor necesario, particularmente en los países en desarrollo.

Para aprender más acerca del impacto de estas intervenciones se realizó un ensayo aleatorizado por 